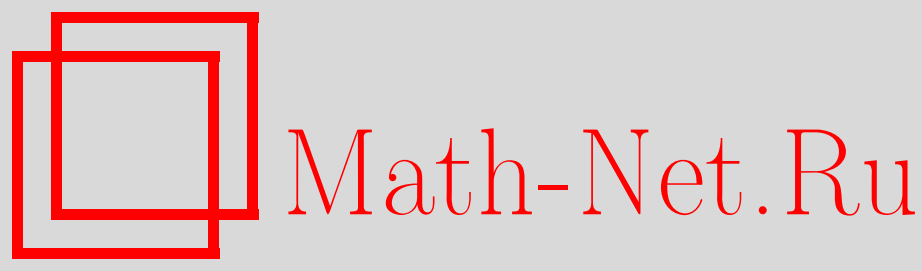

В. Ч. Жуковский, Е. А. Степанов, Генерация массы фермионов и индуцированный ток в низкоразмерных моделях с нетривиальной топологией, ТМФ, 2015, том 182, номер 2, 294-314

DOI: https://doi.org/10.4213/tmf8726

Использование Общероссийского математического портала Math-Net.Ru подразумевает, что вы прочитали и согласны с пользовательским соглашением http: //www . mathnet.ru/rus/agreement

Параметры загрузки:

IP : 3.85 .5 .30

26 апреля 2023 г., 15:58:31

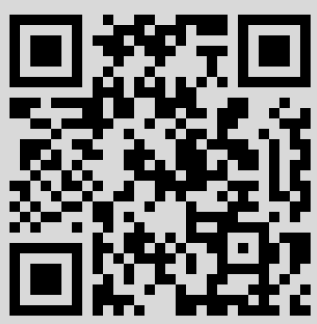




\title{
ГЕНЕРАЦИЯ МАССЫ ФЕРМИОНОВ И ИНДУЦИРОВАННЫЙ ТОК В НИЗКОРАЗМЕРНЫХ МОДЕЛЯХ С НЕТРИВИАЛЬНОЙ ТОПОЛОГИЕЙ
}

\begin{abstract}
Изучен процесс динамического образования массы фермионов в трехмерной модели при взаимодействии двух типов фермионов, существующих в трехмерии и на двумерной бране, с учетом воздействия калибровочного поля $A_{3}$. Исследована модель нанотрубки с размерностью $\mathbb{R}^{2} \times S^{1}$. Установлено, что в выражение для фермионной щели наряду с динамическим вкладом от конденсата $m$ из-за четырехфермионного взаимодействия входит также кинематический вклад фаза Ааронова-Бома, рассчитанная из условия минимума эффективного потенциала модели. Продемонстрирована возможность генерации индуцированного тока за счет эффекта вакуумной поляризации с учетом роли нетривиальной топологии задачи.
\end{abstract}

Ключевые слова: киральный конденсат, модель Калуцы-Клейна, проблема иерархии масс, низкоразмерные системы.

DOI: $10.4213 / \operatorname{tmf} 8726$

\section{1. ВВЕДЕНИЕ}

Одной из физических проблем, решение которой приходится искать за рамками Стандартной модели, является проблема иерархии масс. Стандартная модель включает в себя три поколения элементарных частиц. Соответствующие частицы из разных поколений взаимодействуют одинаково, но массы их отличаются на порядки. Одним из возможных решений данной проблемы является модель Калуцы-Клейна, в которой пространство-время может иметь более четырех измерений. Оригинальной идеей Калуцы и Клейна является то, что дополнительное пятое измерение компактифицировано для описания физических процессов в четырехмерном пространстве-времени нашего мира [1], [2]. В таком случае при изучении обычных физических явлений в пределе малого радиуса компактификации $R \rightarrow 0$ пространство-время выглядит как четырехмерное. Основной стимул для рассмотрения пространства как многомерного дают теории, которые включают в себя гравитацию, например

* Физический факультет, Московский государственный университет им. М. В. Ломоносова, Москва, Россия. E-mail: zhukovsk@phys.msu.ru 
теория струн. Почти все эти теории формулируются в пространстве-времени с числом измерений больше четырех. Многие исследования в этой области указывают на то, что масштаб компактификации должен быть порядка планковского. На планковских масштабах $l \sim 10^{-33}$ см с соответствующей энергией $M_{\mathrm{Pl}} \sim 10^{19}$ ГэВ обнаружение дополнительных измерений на данный момент невозможно, поскольку даже на самом современном ускорителе LHC (Большом адронном коллайдере) достигаются энергии порядка 8 ТэВ (в настоящее время он модернизируется с тем, чтобы достигать энергии порядка 13-14 ТэВ), что много меньше требуемых энергий.

Однако дальнейшее развитие теории показало, что с помощью модели Калуцы-Клейна можно получить массы, много меньшие чем $m=1 / R$. Генерация массы может осуществляться и с помощью юкавской связи четырехмерных скаляров с компонентой калибровочного поля $A_{5}$ из высшего измерения. Поле $A_{5}$ в четырехмерии нарушает калибровочную и киральную симметрии и играет роль хиггсовского поля [3]. Такая генерация массы получила название механизма Хосотани [4].

В последние годы наряду с моделями с дополнительными измерениями развивается и другая область, вызывающая огромный интерес. Это теории с небольшим количеством пространственно-временных измерений (так называемые низкоразмерные модели; см., например, [5]-[8], а также [9]-[11] и указанную там литературу). Так в 1979 году в работе [12] при исследовании линейных полимеров выяснилось, что описание модели полимерной цепочки совпадает в основном с уже известными одномерными моделями квантованных полей. В случае двух пространственных размерностей теория поля давно признана важной для понимания некоторых физических явлений, которые могут быть приближенно рассмотрены как плоские. Особенный интерес к двумерным моделям возникает в физике конденсированного вещества, в рамках которой было открыто большое число важных новых явлений.

Примером такой двумерной модели является графен - плоский одноатомный слой углерода, который обладает целым рядом необычных характеристик [13]-[15]. В ряде недавних исследований [16]-[18] были открыты аномальный эффект Холла, необычные свойства проводимости и ряд других интересных характеристик этого материала. Особенностью эффекта Холла в графене является то, что его можно наблюдать даже при комнатной температуре [19] (при значениях магнитного поля больше $20 \mathrm{~T})$.

В описании графена поведение электронов эффективно подчиняется уравнению Дирака [20]-[23], и в таком случае удобно рассматривать эту задачу в рамках хорошо известных методов квантовой механики и квантовой теории поля для фермионов в пространстве размерности $2+1$. В частности, таким образом была предложена псевдопотенциальная модель для описания прохождения дираковских электронов через линейные дефекты в графене [24]. Следует отметить, что эффективные теоретико-полевые модели Гросса-Невё [25] и Намбу-Йона-Лазинио [26]-[29] также хорошо подходят для решения задач подобного рода. В таких плоских системах модель Гросса-Невё обычно используется для исследования свойств симметрии, нарушения киральной симметрии [30], а также для задач генерации массы фермионов [31].

Другим примером модели с малым числом пространственных измерений является полиацетилен. Интерес к этой модели вызван рядом причин. С экспериментальной точки зрения появляется возможность создания нанополупроводниковых устройств 
из этого материала, а с теоретической точки зрения эту модель можно представить как одномерную модель графена. В работе [32] была рассмотрена модель полиацетилена в рамках модели Гросса-Невё с размерностью $1+1$ и были продемонстрированы нарушение киральной симметрии и генерация фермионной массы.

Низкоразмерные модели с электромагнитными полями и нетривиальной топологией, подобные двумерной модели графена и фуллерена, рассматривались в недавних работах [33]-[35]. Подобная проблема также обсуждалась в работе [36] при исследовании модели углеродной нанотрубки (см. также [37]-[39]). В модели [36] исследовалась генерация массы фермионов под влиянием внешнего магнитного поля Ааронова-Бома [40]. При этом под действием внешнего магнитного поля может происходить поляризация вакуума (см., например, [41], [42]), что приводит к появлению индуцированного тока. Так, в работе [42] была рассмотрена поляризация вакуума в графене в поле тонкого соленоида с возникновением индуцированного тока. В настоящее время моделям с низким числом пространственных измерений продолжает уделяться много внимания. В работе [43] была предложена модель, в которой присутствуют два типа фермионов, одни существуют в пятимерном пространстве-времени и взаимодействуют с другими фермионами, существующими на четырехмерной бране. Подобное поведение можно описать с помощью четырехфермионного взаимодействия, эффективно возникающего при обмене гравитонами, что ведет к генерации динамической массы. В работе [44] модель из работы [43] была рассмотрена в пятимерии, где высшая размерность была компактифицирована по окружности с радиусом компактификации $R$ и, кроме того, были добавлены периодические и антипериодические граничные условия для фермионов. Модель была расширена введением постоянного калибровочного поля $A_{5}$, находящегося в пятимерном пространстве, для исследования нарушения киральной симметрии при четырехфермионном взаимодействии и получения динамической массы для легких фермионов.

В настоящей работе исследуются низкоразмерные модели в размерности $2+1$ с компактификацией “дополнительного” измерения. В таком случае получается, например, плоская модель с браной размерностью $1+1$ (с пространственной размерностью 1), т. е. с нитью. В рамках модели Гросса-Невё с двумя типами фермионов исследуется влияние параметров модели, таких как калибровочное поле $A_{3}$, параметр фазового смещения фермионов $\alpha$ и радиус компактификации $R$, на генерацию массы фермионов. Рассматривается также модель нанотрубки, в рамках которой исследуются влияние эффекта Ааронова-Бома на генерацию фермионной массы и возникновение индуцированного тока за счет поляризации вакуума в присутствии реального магнитного поля.

\section{2. ГЕНЕРАЦИЯ ФЕРМИОННОЙ МАССЫ ПОД ВЛИЯНИЕМ КАЛИБРОВОЧНОГО ПОЛЯ В МОДЕЛИ С $2+1$ ИЗМЕРЕНИЯМИ}

2.1. Модель. Рассмотрим трехмерную фермионную модель, содержащую два типа фермионных полей $\Psi$ и $L$, и калибровочное поле $A_{M}$ в трехмерном пространстве-времени [45]. $\Psi$-фермионы существуют в трехмерном пространстве, а $L$-фермионы - на двумерной бране. Лагранжиан модели аналогичен лагранжиану для 
пятимерной модели [43]:

$$
\mathcal{L}^{(3)}=\bar{\Psi} i \gamma^{M} D_{M} \Psi+\left[\bar{L} i \gamma^{\mu} D_{\mu} L+g^{2}\left(\bar{\Psi} \gamma^{M} L\right)\left(\bar{L} \gamma_{M} \Psi\right)\right] \delta\left(x^{3}\right),
$$

где $M=1,2,3, \mu=1,2, D_{M}=\partial_{M}-i e A_{M}$. Здесь используются метрика $(+,-,-)$ и $\gamma$-матрицы, заданные следующим образом:

$$
\gamma^{1}=\left(\begin{array}{cc}
1 & 0 \\
0 & -1
\end{array}\right), \quad \gamma^{2}=\left(\begin{array}{cc}
0 & i \\
i & 0
\end{array}\right), \quad \gamma^{3}=\left(\begin{array}{cc}
0 & 1 \\
-1 & 0
\end{array}\right) .
$$

Представим калибровочное поле как конденсат со средним значением компонент $\left\langle A_{3}\right\rangle=$ const $\neq 0$ и $\left\langle A_{1}\right\rangle=\left\langle A_{2}\right\rangle=0$. Тогда лагранжиан примет вид

$$
\mathcal{L}^{(3)}=\bar{\Psi} \gamma^{3} e A_{3} \Psi+\bar{\Psi} i \gamma^{M} \partial_{M} \Psi+\left[\bar{L} i \gamma^{\mu} \partial_{\mu} L+g^{2}\left(\bar{\Psi} \gamma^{M} L\right)\left(\bar{L} \gamma_{M} \Psi\right)\right] \delta\left(x^{3}\right) .
$$

Проведем преобразование Хаббарда-Стратоновича, введя вспомогательное поле $\sigma_{M}$. Далее используем приближение среднего поля, заменяя реальное поле его средним значением, тогда $\left\langle\sigma_{\mu}\right\rangle=0,\left\langle\sigma_{3}\right\rangle=\sigma_{3}=-\sigma$. Совершив киральный поворот [46] $\Psi \rightarrow e^{\pi \gamma_{3} / 4} \Psi, L \rightarrow e^{\pi \gamma_{3} / 4} L$, получим

$$
\mathcal{L}^{(3)}=\bar{\Psi} i \not \partial \Psi-\bar{\Psi} e A_{3} \Psi-i \bar{\Psi} \partial_{3} \Psi+\left[\bar{L} i \not \partial L-|\sigma|^{2}+(g \sigma \bar{\Psi} L+\text { э.c. })\right] \delta\left(x^{3}\right) .
$$

Компактифицируем третью размерность по окружности радиуса $R$ и зададим дополнительный параметр - фазовое смещение фермионов $\alpha$ :

$$
\Psi=N \sum_{n=-\infty}^{+\infty} \Psi_{n}\left(x_{\mu}\right) e^{i x_{3}(n+\alpha) / R},
$$

тогда двумерный лагранжиан примет вид

$$
\begin{aligned}
\mathcal{L}^{(2)}=\int_{0}^{2 \pi R} d x^{3} \mathcal{L}^{(3)}= & \sum_{n=-\infty}^{+\infty} \bar{\Psi}_{n} i \not \partial \Psi_{n}+\sum_{n=-\infty}^{+\infty}\left(\frac{n+\alpha}{R}-e A_{3}\right) \bar{\Psi}_{n} \Psi_{n}+ \\
& +\bar{L} i \not \partial L-|\sigma|^{2}+\left(m \sum_{n=-\infty}^{+\infty} \bar{\Psi}_{n} L+\text { э.c. }\right),
\end{aligned}
$$

где $m=N g \sigma, N=1 / \sqrt{2 \pi R}-$ нормировочная константа.

2.2. Спектр масс. Перейдем к матричному представлению для фермионных полей

$$
(\Psi)^{\mathrm{T}}=\left(L, \Psi_{0}, \Psi_{1}, \Psi_{-1}, \Psi_{2}, \Psi_{-2}, \ldots\right) .
$$

Тогда массовая матрица запишется в виде $\left(e A_{3} \equiv a\right)$

$$
M=\left(\begin{array}{cccccc}
0 & m^{*} & m^{*} & m^{*} & m^{*} & \ldots \\
m & \frac{\alpha}{R}-a & 0 & 0 & 0 & \ldots \\
m & 0 & \frac{\alpha+1}{R}-a & 0 & 0 & \ddots \\
m & 0 & 0 & \frac{\alpha-1}{R}-a & 0 & \ddots \\
m & 0 & 0 & 0 & \frac{\alpha+2}{R}-a & \ddots \\
\vdots & \vdots & \ddots & \ddots & \ddots & \ddots
\end{array}\right) .
$$


В результате получим уравнение на собственные значения:

$$
\begin{aligned}
& \operatorname{det}(M-\lambda I)=\left\{\prod_{j=1}^{\infty}\left[\left(\lambda+a-\frac{\alpha}{R}\right)^{2}-\left(\frac{j}{R}\right)^{2}\right]\right\} \times \\
& \quad \times\left[\lambda\left(\lambda+a-\frac{\alpha}{R}\right)-|m|^{2}-2\left(\lambda+a-\frac{\alpha}{R}\right)^{2}|m|^{2} \sum_{l=1}^{+\infty} \frac{1}{(\lambda+a-\alpha / R)^{2}-(l / R)^{2}}\right]=0 .
\end{aligned}
$$

K нетривиальным решениям приводит равенство нулю только второй скобки, что дает

$$
\lambda R=m^{2} R^{2} \pi \operatorname{ctg}\left(\pi R\left(\lambda+a-\frac{\alpha}{R}\right)\right) .
$$

В случае, когда аргумент котангенса является малым параметром, из предыдущей формулы имеем

$$
\pi \lambda R=(\pi|m| R)^{2} \frac{1}{\pi R(\lambda+a-\alpha / R)},
$$

откуда получаем

$$
\lambda=\frac{\alpha-a R \pm \sqrt{(a R-\alpha)^{2}+4|m|^{2} R^{2}}}{2 R} .
$$

Из уравнения (12) видно, что генерируемая масса зависит от радиуса компактификации, параметра фазового смещения $\alpha$ и калибровочного поля $a$. Таким образом, мы можем получать различные значения массы, варьируя эти параметры. Заметим, что при условиях $a=0$ и $\alpha=0$ получаем $\lambda= \pm|m|$. Условие малости аргумента котангенса в таком случае переписывается в виде $\lambda \ll 1 / R$, откуда следует, что и $|m| \ll 1 / R$. Таким образом, мы получили массу для легких фермионов $\lambda= \pm|m|$, много меньшую массы мод Калуцы-Клейна для фермионов в трехмерии. Следовательно, данный результат может рассматриваться как указание на одну из возможностей обоснования проблемы иерархии масс (см. [45]).

2.3. Эффективный потенциал модели. Запишем эффективный лагранжиан модели в матричном виде:

$$
\mathcal{L}_{\mathrm{eff}}^{(2)}=\bar{\Psi} i \not \partial \Psi+\bar{\Psi} M \Psi-|\sigma|^{2} .
$$

Производящий функционал нашей системы дается формулой

$$
Z=\int[\mathcal{D} \bar{\Psi}][\mathcal{D} \Psi][\mathcal{D} \sigma]\left[\mathcal{D} \sigma^{*}\right] \exp \left[i \int d^{2} x \mathcal{L}^{(2)}\right]
$$

Интегрируя по фермионным полям, получаем

$$
Z=\int[\mathcal{D} \sigma]\left[\mathcal{D} \sigma^{*}\right] \exp \left[-i \int d^{2} x V_{\mathrm{eff}}(\sigma)\right]
$$

где эффективный потенциал определяется стандартным образом:

$$
V_{\text {eff }}=|\sigma|^{2}-\int \frac{d^{2} k}{(2 \pi)^{2}} \ln \operatorname{det}(M+I \not k) .
$$




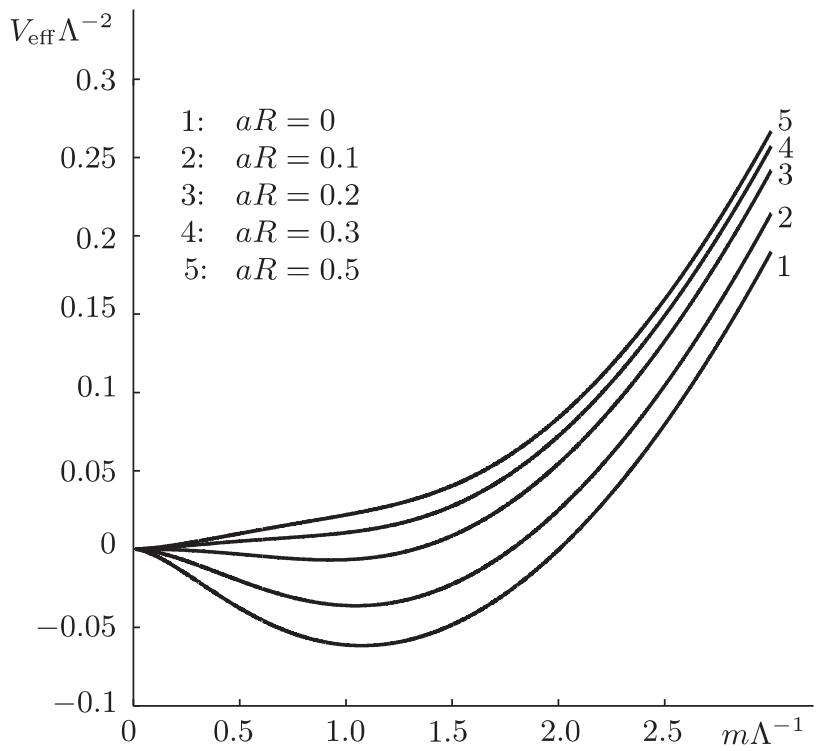

Рис. 1. Зависимость эффективного потенциала от $m \Lambda^{-1}$ при нулевом значении параметра фазового смещения $\alpha$ и при $R \Lambda=0.1$.

Отсюда с помощью формулы (9) найдем

$$
\begin{aligned}
V_{\mathrm{eff}}(\sigma, a)=|\sigma|^{2} & -\frac{1}{4 \pi} \int_{0}^{\Lambda} d x x \ln \left\{x^{2}[\operatorname{ch}(2 \pi R x)-\cos (2 \pi R a)]+\right. \\
& \left.+2 \pi R x|m|^{2} \operatorname{sh}(2 \pi R x)+\left(\pi R|m|^{2}\right)^{2}[\operatorname{ch}(2 \pi R x)+\cos (2 \pi R a)]\right\}
\end{aligned}
$$

где мы ввели параметр обрезания $\Lambda$, поскольку интеграл расходится на верхнем пределе. В предыдущей формуле для сокращения выражений мы положили $\alpha=0$, так как включение конечного значения фазы $\alpha$, входящей аддитивно с $a R$, может быть легко учтено в конечном результате. Интеграл (17) в критической точке $m=0$ $(\sigma=0)$ можно преобразовать к следующему виду:

$$
\left.V_{\mathrm{eff}}(\sigma, a)\right|_{\sigma=0}=-\frac{1}{4 \pi} \int_{0}^{\Lambda} d x x \ln [\operatorname{ch}(2 \pi R x)-\cos (2 \pi R a)]
$$

График поведения эффективного потенциала при $\alpha=0$ изображен на рис. 1 . Как видно, эффективный потенциал имеет экстремум $(m R \simeq 0.1)$ при значениях поля $a R=0$ и экстремум в нуле при $a R=1 / 2$.

2.4. Критическая константа связи. Запишем уравнение щели, $\partial V_{\text {eff }} / \partial \sigma=0$ :

$$
1-\int_{0}^{\Lambda} \frac{d k k}{4 \pi} \frac{\left(k \operatorname{sh}(\pi k R)+\left(g^{2} \sigma^{2} / 2\right) \operatorname{ch}(\pi k R)\right) g^{2} \operatorname{ch}(\pi k R)-\left(g^{4} \sigma^{2} / 2\right) \sin ^{2}(\pi A)}{\left(k \operatorname{sh}(\pi k R)+\left(g^{2} \sigma^{2} / 2\right) \operatorname{ch}(\pi k R)\right)^{2}+\left(k^{2}-g^{4} \sigma^{4} / 4\right) \sin ^{2}(\pi A)}=0
$$




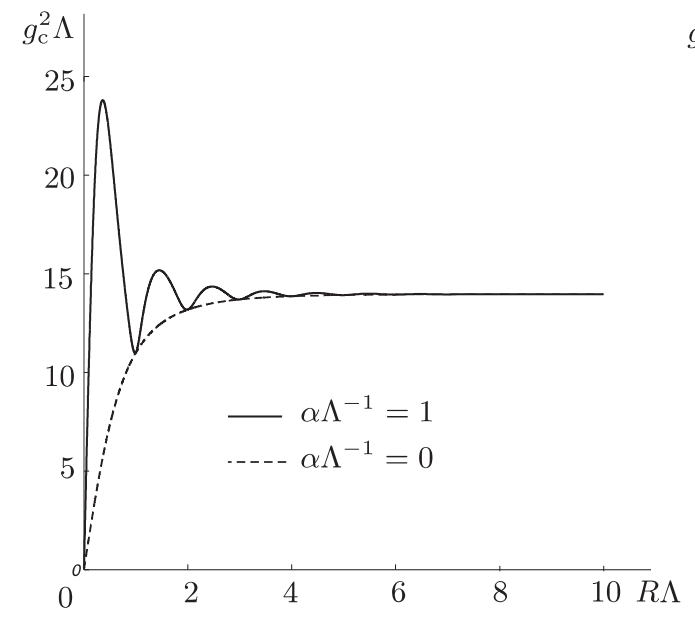

a

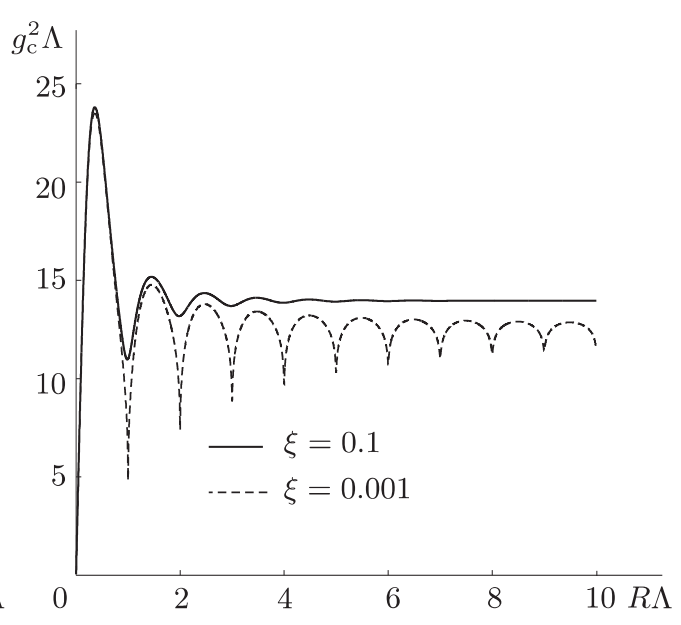

б

Рис. 2. Зависимость $g_{\mathrm{c}}^{2} \Lambda$ от $R \Lambda$ при $\alpha=0, \xi=0.1$ и при различных параметрах поля $a(\mathrm{a})$; та же зависимость при $\alpha \Lambda^{-1}-=1, \alpha=0$ и при различных параметрах нижнего обрезания интеграла $\xi(б)$.

где $A=\alpha-a R$. Вычислим критическую константу связи, которая определяется из условия $|\sigma|=0$ :

$$
1-\int_{\xi}^{\Lambda} \frac{d k}{4 \pi} \frac{g_{c}^{2} \operatorname{sh}(\pi k R) \operatorname{ch}(\pi k R)}{\operatorname{sh}^{2}(\pi k R)+\sin ^{2}(\pi A)}=0,
$$

где обрезание $\xi$ введено, поскольку интеграл расходится на нижнем пределе при $A \rightarrow 0$. Вычисляя получившийся интеграл, получаем

$$
g_{\mathrm{c}}^{2} \Lambda=8 \pi^{2} R \Lambda\left\{\ln \left[\frac{\operatorname{ch}(2 \pi R \Lambda)-\cos (2 \pi R a-2 \pi \alpha)}{\operatorname{ch}(2 \pi R \xi)-\cos (2 \pi R a-2 \pi \alpha)}\right]\right\}^{-1}
$$

При $R \Lambda \rightarrow \infty$ имеем

$$
g_{\mathrm{c}}^{2} \rightarrow \frac{4 \pi}{\Lambda}
$$

что соответствует поведению критической константы в трехмерном пространстве [47].

Переходя для удобства к безразмерным переменным $R \Lambda \rightarrow R, g^{2} \Lambda \rightarrow g^{2}, \xi / \Lambda \rightarrow \xi$, получим

$$
g_{\mathrm{c}}^{2}=8 \pi^{2} R\left\{\ln \left[\frac{\operatorname{ch}(2 \pi R)-\cos (2 \pi R a / \Lambda-2 \pi \alpha)}{\operatorname{ch}(2 \pi R \xi)-\cos (2 \pi R a / \Lambda-2 \pi \alpha)}\right]\right\}^{-1} .
$$

На рис. $2 б$ и рис. За изображены зависимости критической константы связи при различных значениях параметра обрезания $\xi$. На рис. 2 а при значении $a \Lambda^{-1}=1$ критическая константа связи ведет себя как затухающая осциллирующая функция, стремящаяся асимптотически к критической константе связи при $a \Lambda^{-1}=0$. На рис. $2 б$ при достаточно малом значении параметра обрезания $\xi=0.001$ график критической константы связи получается негладким и на нем имеются особенности - 

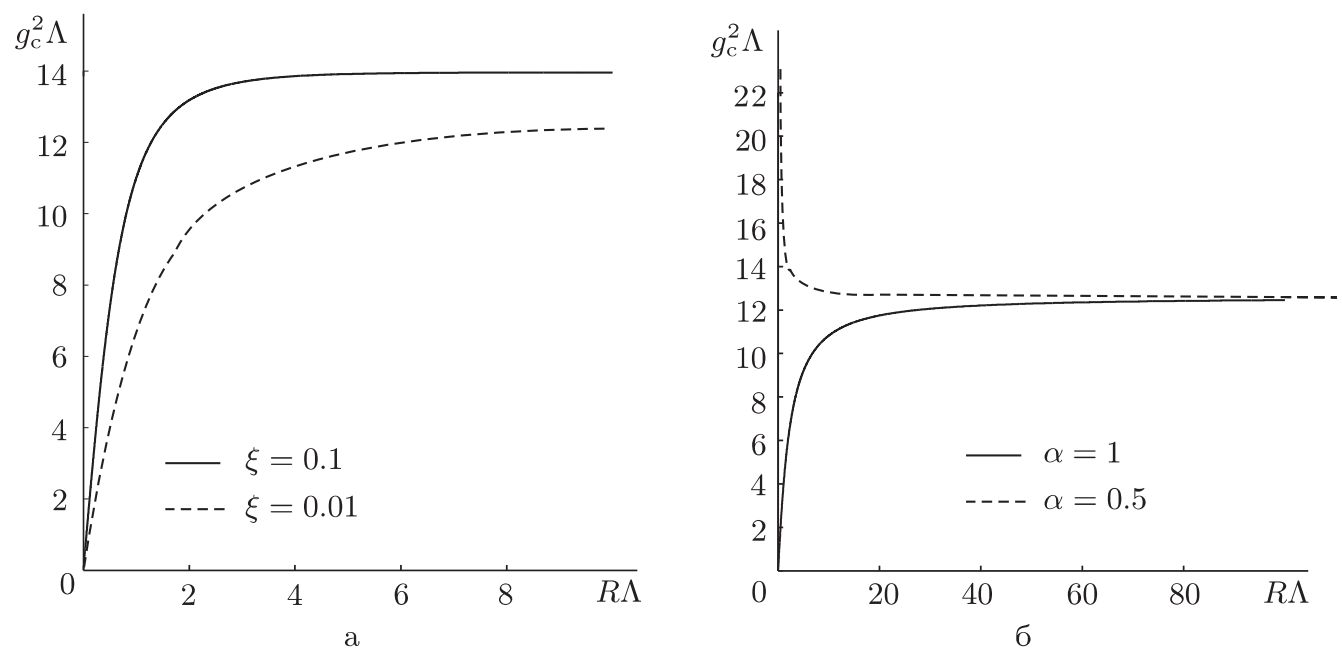

Рис. 3. Поведение $g_{\mathrm{c}}^{2} \Lambda$ при $a \Lambda^{-1}=0, \alpha=0$ и при различных параметрах обрезания $\xi(\mathrm{a})$; та же зависимость при значении параметра обрезания $\xi=0$, $a \Lambda^{-1}=0$ и при различных граничных условиях: периодических $(\alpha=1)$ и антипериодических $(\alpha=0.5)$ (б).

острия. При более реалистическом значении этого параметра $\xi=0.1$ график оказывается похожим на поведение константы в пятимерии [44]. На рис. $3 б$ параметр обрезания выбран стремящимся к нулю $(\xi \rightarrow 0)$, его роль здесь выполняет параметр фазового смещения $\alpha$.

2.5. Динамическое поле $a$. Теперь рассмотрим случай, когда $a$ - динамическая переменная (см. [45]). Тогда экстремум эффективного потенциала дается уравнением щели $\partial V_{\text {eff }} / \partial a=0$ для поля $a$ :

$$
\int_{0}^{\Lambda} \frac{d k k \sin (2 \pi(\alpha-a R)) \pi R\left(k^{2}-m^{4} \pi^{2} R^{2}\right)}{4 \pi\left(k \operatorname{sh}(\pi k R)+m^{2} \pi R \operatorname{ch}(\pi k R)\right)^{2}+\left(k^{2}-m^{4} \pi^{2} R^{2}\right) \sin ^{2}(\pi(\alpha-a R))}=0
$$

откуда находим, что экстремум имеет место при $\sin (2 \pi(\alpha-a R))=0$ или

$$
\alpha-a R=\frac{n}{2}
$$

где $n$ - целые числа. Тогда $a=(2 \alpha-n) / 2 R$, что эквивалентно решению $a=\alpha / R$ при четных $n=2 k$ и $a=(2 \alpha-1) / 2 R$ при нечетных $n=2 k+1$. В частности, при периодических граничных условиях $(\alpha=0) a=0$ и $a=-1 / 2 R$, при антипериодических $(\alpha=1 / 2) a=0$ и $a=1 / 2 R$.

При четных значениях $n, \cos (2 \pi R a-2 \pi \alpha)=1$, критическая константа связи имеет вид

$$
g_{\mathrm{c}}^{2}=8 \pi^{2} R\left\{\ln \left[\frac{\operatorname{ch}(2 \pi R)-1}{\operatorname{ch}(2 \pi R \xi)-1}\right]\right\}^{-1}
$$


При нечетных значениях $n, \cos (2 \pi R a-2 \pi \alpha)=-1$, критическая константа связи имеет вид

$$
g_{\mathrm{c}}^{2}=8 \pi^{2} R\left\{\ln \left[\frac{\operatorname{ch}(2 \pi R)+1}{\operatorname{ch}(2 \pi R \xi)+1}\right]\right\}^{-1} .
$$

Оценим генерируемую массу при критических значениях поля $a$.

Для четных значений $n$ имеем $a-\alpha / R=0$. В этом случае получим

$$
\lambda R=\pi|m R|^{2} \operatorname{ctg}(\pi R \lambda) .
$$

Нас интересуют легкие фермионы с массами $\lambda \ll 1 / R$, в этом случае $\lambda=|m|$.

Для нечетных значений $n$ имеем $a-\alpha / R=-1 / 2 R$. В этом случае получим

$$
\lambda R=\pi|m R|^{2} \operatorname{ctg}\left(\pi R \lambda-\frac{\pi}{2}\right) .
$$

Для $\pi(R \lambda-1 / 2) \ll 1$ получим

$$
\lambda R=\frac{|m R|^{2}}{\lambda R-1 / 2}
$$

или

$$
\lambda R=\frac{1}{4}+\sqrt{\frac{1}{16}+|m R|^{2}} \approx \frac{1}{2}+O\left(|m R|^{2}\right) .
$$

Таким образом, $\lambda \approx 1 / 2 R=a$. Тем самым мы получаем массу, меньшую, чем для мод Калуцы-Клейна $\lambda_{n}=n / R$.

2.6. Асимптотическое поведение константы связи. Рассмотрим поведение константы связи при предельных значениях радиуса компактификации $R$ (см. [48]). Вернемся к уравнению щели (19), положив $A=0$ и выразив все величины через $m$ $(\sigma=(m / g) \sqrt{2 \pi R})$ :

$$
1-\int_{0}^{\Lambda} \frac{k d k}{4 \pi} \frac{g^{2} \operatorname{ch}(\pi k R)}{k \operatorname{sh}(\pi k R)+m^{2} \pi R \operatorname{ch}(\pi k R)}=0 .
$$

Если $R$ мало, т. е. $m R \ll 1$, и в то же время $\Lambda R \gg 1$ (т. е. считаем, что $g^{2} / \pi R=$ const $=g_{2}-$ константа связи в двумерии), $k$ дает основной вклад при $k \sim m$. Этот случай соответствует компактификации $3 D \rightarrow 2 D$. Следовательно, $\operatorname{ch}(\pi k R) \approx 1+$ $(\pi k R)^{2} / 2$ и $\operatorname{sh}(\pi k R) \approx \pi k R$, тогда получаем

$$
1-\int_{0}^{\Lambda} \frac{k d k}{4 \pi} \frac{g^{2}\left(1+(\pi k R)^{2} / 2\right)}{k^{2} \pi R+\pi R m^{2}\left(1+(\pi k R)^{2} / 2\right)}=0,
$$

откуда имеем

$$
g^{2}=8 \pi\left(\pi R+m^{2} \pi^{3} R^{3}\right)\left\{\ln \left[\frac{\Lambda^{2}+\Lambda^{2} m^{2} \pi^{2} R^{2}}{2 m^{2}}+1\right]\right\}^{-1}
$$



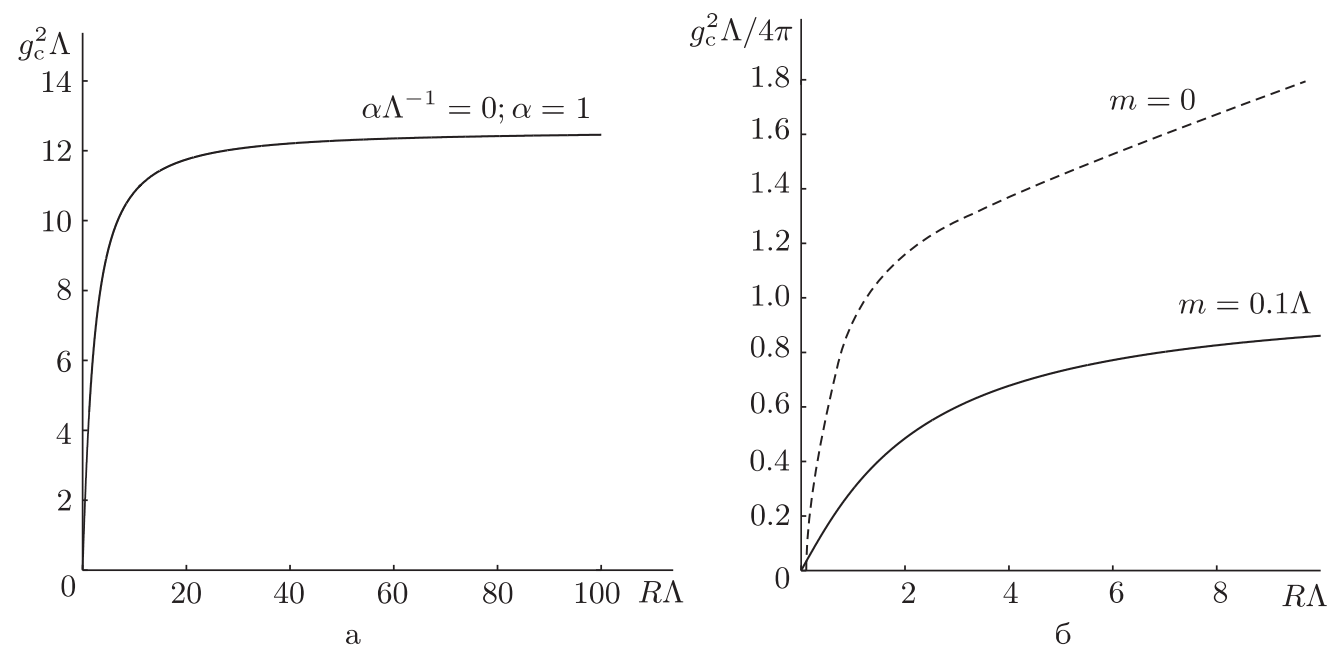

Рис. 4. Поведение $g_{\mathrm{c}}^{2} \Lambda$ при значениях $\alpha-a R=n / 2$, при которых эффективный потенциал $V_{\text {eff }}$ имеет экстремум (а). Поведение $g^{2} \Lambda$ при различных значениях $m / \Lambda$ (б).

Поскольку $m R \ll 1$, получим

$$
\frac{g^{2}}{4 \pi R}=g_{2}=\frac{2 \pi}{\ln \left(\Lambda^{2} / m^{2}\right)},
$$

или

$$
m=\Lambda e^{-\pi / g_{2}},
$$

что согласуется с результатом [47]

$$
m=\frac{2}{\beta} e^{-\pi / g}
$$

для двумеризованной модели с точностью до очевидной замены обозначений. Сравнивая эффективный потенциал задачи [47]

$$
V_{\mathrm{eff}}=-\frac{1}{(2 \pi)^{2}} \int d^{2} k \ln \left(k^{2}+\phi^{2}\right)+\frac{1}{2 g} \phi^{2}
$$

с нашим результатом, очевидно, находим, что коэффициент $2 / \beta$ выбран как параметр обрезания интеграла (что соответствует $\Lambda$ в нашей модели), $g$ в модели [47] соответствует $g^{2} / 4 \pi R$ нашей модели, а $\phi$ соответствует $m / 2$ нашей модели. График зависимости $g^{2} \Lambda$ от $R \Lambda$ при различных значениях параметра $m / \Lambda$ показан на рис. $4 б$. В пределе больших $R \Lambda$ верхняя кривая стремится к бесконечности, а нижняя стремится к $4 \pi$. Из графика видно, что при малом радиусе компактификации $(R<1)$ значение констант связи при разных $m$ различно, тогда как при $R \rightarrow \infty$ критическая константа связи стремится к своему асимптотическому значению (22). Если рассмотреть константу связи, лежащую немного выше критической $(m=0.1 \Lambda)$, то при малых значениях $R$ мы можем получить различные малые значения констант 
связи и, следовательно, различные малые массы. Таким образом, в рамках данной модели существует возможность объяснить иерархию масс разных поколений частиц.

Зависимость константы связи от малого радиуса компактификации также дается формулой (35).

При $R \rightarrow \infty, \operatorname{sh}(\pi k R) \rightarrow e^{\pi k R / 2}$ и $\operatorname{ch}(\pi k R) \rightarrow e^{\pi k R / 2}$, т. е. это величины одного порядка, тогда можно воспользоваться логарифмическим приближением. Действительно, сделаем замену $\pi k R \rightarrow x$ :

$$
\int_{m^{2} \pi^{2} R^{2}}^{\pi \Lambda R} \frac{d \operatorname{sh} x x}{x \operatorname{sh} x+m^{2} \pi^{2} R^{2} \operatorname{ch} x}=\frac{4 \pi^{2} R}{g^{2}} .
$$

Основной вклад в знаменатель при $R \rightarrow \infty$ дает слагаемое $x \operatorname{sh} x$, поскольку в случае больших $R$ имеем $x \gg 1, g=$ const и $m^{2} R=$ const $=M_{3}$. Таким образом, $x \gg m^{2} \pi^{2} R^{2}$, и основной вклад в интеграл дается в пределах от $m^{2} \pi^{2} R^{2}$ до $\pi \Lambda R$. Используя данную асимптотику, получим

$$
g^{2}=\frac{4 \pi}{\Lambda-m^{2} R \pi}
$$

или

$$
m^{2} R=M_{3}=\frac{\Lambda}{\pi}-\frac{4}{g^{2}}=4\left(\frac{1}{g_{\mathrm{c}}^{2}}-\frac{1}{g^{2}}\right),
$$

что также согласуется с результатом [47] для трехмерного предела.

2.7. Связь параметра обрезания $\xi$ с конденсатом $m$. В полученном результате для критической константы связи положим $\alpha=0$, тогда получим

$$
g_{\mathrm{c}}^{2}=8 \pi^{2} R\left\{\ln \left[\frac{\operatorname{ch}(2 \pi R)-\cos (2 \pi R a / \Lambda)}{\operatorname{ch}(2 \pi R \xi / \Lambda)-\cos (2 \pi R a / \Lambda)}\right]\right\}^{-1} .
$$

При $R \rightarrow 0$ имеем

$$
g_{\mathrm{c}}^{2}=8 \pi^{2} R\left\{\ln \left[\frac{\Lambda^{2}+\xi^{2}}{\xi^{2}+a^{2}}+1\right]\right\}^{-1} .
$$

Устремляя $\xi \rightarrow 0$ (рассматриваем инфракрасный предел) и проводя сравнение с выражением для асимптотики константы связи, получим $a^{2} \sim 2 m^{2}$, откуда видно, что поле $a$ в выражении для критической константы связи играет роль конденсата $m$ в выражении для обычной константы связи. Если же положить $a \rightarrow 0$, то получим $\xi^{2} \sim 2 m^{2}$, откуда следует, что роль параметра обрезания в обычной константе связи играет конденсат $m$ (см. [48]).

При $R \rightarrow \infty$ получаем

$$
g_{\mathrm{c}}^{2}=\frac{4 \pi}{\Lambda-\xi}
$$

Поскольку $\xi$ - малый параметр инфракрасного обрезания, то он мал по сравнению с $\Lambda$, тогда в пределе $R \rightarrow \infty$

$$
g_{\mathrm{c}}^{2}=\frac{4 \pi}{\Lambda}
$$

что согласуется с результатом (22). 


\section{3. ВЛИЯНИЕ МАГНИТНОГО ПОТОКА НА ПОВЕДЕНИЕ ФЕРМИОНОВ В ДВУМЕРНОЙ МОДЕЛИ С НЕТРИВИАЛЬНОЙ ТОПОЛОГИЕЙ}

Исследуем теперь модель, аналогичную той, которая рассматривалась в предыдущем разделе, в рамках рассмотрения моделей с малым числом измерений, включающих в себя реальное поле, аналогично двумерным моделям графена с нетривиальной топологией [33], [34] и фулеренов [35]. В этих моделях рассматривается влияние реального магнитного поля на поведение фермионов. Существование такого магнитного поля ведет к редукции числа измерений на две единицы $D+1 \rightarrow(D-2)+1$, следовательно, все эти модели имеют малое число измерений. Реальное магнитное поле в данной задаче создает теперь только магнитный поток, что соответствует задаче Ааронова-Бома [40].

В 1959 году Аароновым и Бомом был предсказан эффект, согласно которому электромагнитное поле в той области, где напряженность электрического поля и индукция магнитного поля равны нулю, может влиять на поведение заряженной частицы на квантовом уровне. Действительно, если в таком случае электромагнитный потенциал не равен нулю, то именно он и оказывает влияние на заряженные частицы. Эффект Ааронова-Бома был проверен экспериментально сначала в 1960 году [49], а потом в 1986 году [50] в опытах со сверхпроводящими материалами. В дальнейшем задачи типа Ааронова-Бома стали широко исследоваться и данное направление получило большое развитие.

Из недавних работ стоит отметить, например, работы [36], [51]. В работе [36] рассматривается модель в размерности $\mathbb{R}^{2} \times S^{1}$, т. е. углеродная нанотрубка. В рамках этой модели исследуется генерация фермионной массы с помощью потенциала поля из компактифицированного измерения (механизм Хосотани [4]), рассчитывается эффективный потенциал модели и с помощью уравнения щели находится выражение для фермионной щели как экстремум эффективного потенциала. Эта щель будет убывать с магнитным полем, и динамическое нарушение симметрии происходит не так, как в большинстве известных случаев. Поскольку в данной задаче магнитное поле направлено параллельно оси цилиндра и не влияет на движущиеся заряженные частицы, а создает лишь магнитный поток, авторы искусственно добавляют к динамическому вкладу в щель кинематический член $2 \pi \phi_{||}$- фазу Ааронова-Бома, где $\phi_{\|}=e A_{y} L / 2 \pi$, и эта полная щель тогда уже будет возрастать с магнитным полем. Эта добавленная фаза была вычислена в работе [51], где была рассмотрена модель размерности $\mathbb{R}^{3} \times S^{1}$ без внешнего магнитного поля. При этих условиях фаза Ааронова-Бома получается из условия наличия ненулевого электромагнитного векторного потенциала, который нельзя свести к нулю выбором калибровки из-за условия периодичности. В этой задаче фаза Ааронова-Бома не является внешним параметром, как в работе [36], а определяется из условия минимума энергии. Авторы выбирают внешнее поле третьей компоненты как $\bar{A}_{\nu}=\Delta \delta_{\nu 3}$, которое подчиняется калибровочному преобразованию $A_{\mu} \rightarrow A_{\mu}-(1 / e) \partial_{\mu} \alpha$, где $\alpha$ удовлетворяет следующим граничным условиям: $\alpha\left(x_{3}+a\right)=\alpha\left(x_{3}\right)+2 \pi l, l \in \mathbb{Z}, a-$ длина дополнительной размерности $(2 \pi R)$. После этого из условия минимума эффективного потенциала можно получить $\bar{A}_{3}=\pi / e a(l=1)$. 
В начале настоящего раздела, как и в работах [36], [51], исследуется влияние магнитного потока на генерацию фермионной массы в модели нанотрубки размерности $\mathbb{R}^{2} \times S^{1}$ с двумя типами фермионов.

Недавно в работе [42] была рассмотрена поляризация вакуума в поле тонкого соленоида и исследовано возникновение индуцированного тока. В качестве применения полученного результата авторы рассмотрели графен в поле соленоида, перпендикулярного плоскости образца.

Ниже в настоящем разделе мы показываем, что ненулевой потенциал $A_{3}$ третьей компоненты поля кроме влияния на генерацию фермионной массы сказывается и на появлении индуцированного тока. Мы продемонстрируем, что индуцированный ток возникает благодаря поляризации вакуума в калибровочном поле, заданном третьей компонентой потенциала $A_{3}$ в $(2+1)$-мерной модели [45] с двумя типами фермионов, сушествующими на бране и во всем объеме образца.

3.1. Вклад фазы Ааронова-Бома в фермионную щель. Рассмотрим модель с лагранжианом, аналогичным лагранжиану раздела 2, но с другой точки зрения. После компактификации третьей координаты будем рассматривать получившуюся модель как нанотрубку с магнитным полем, направленным вдоль оси нанотрубки (см. [45]). Такое магнитное поле не будет влиять на поведение электронов, но ненулевой потенциал $A_{3}$, направленный вдоль окружности радиуса $R$ (т. е. вдоль компактифицированной третьей координаты), будет вносить свой вклад в генерацию фермионной массы. Постановка нашей задачи отличается от случаев, рассмотренных в работах [36], [51], наличием четырехфермионного взаимодействия в пространстве размерности $\mathbb{R}^{2} \times S^{1}$ и фазового параметра $\alpha$, соответствующего периодическому (антипериодическому) граничному условию. Граничные условия по третьей координате имеют следующий вид:

$$
\Psi\left(x_{\mu}, x_{3}+2 \pi R\right)=\Psi\left(x_{\mu}, x_{3}\right)
$$

а компактификация дополнительного измерения задается условием

$$
\Psi\left(x_{\mu}, x_{3}\right)=N \sum_{n=-\infty}^{\infty} \Psi_{n}\left(x_{\mu}\right) e^{i x_{3}(n+\alpha) / R} .
$$

Лагранжиан нашей модели инвариантен относительно калибровочных преобразований $A_{\mu} \rightarrow A_{\mu}+(1 / e) \partial_{\mu} \beta$ и $\Psi \rightarrow e^{i \beta} \Psi$, где $\beta=x_{3} n / R, n \in \mathbb{Z}$. Поскольку наш параметр поля $a$ с точностью до $e$ аналогичен $\Delta$ из работы [51], то, находя экстремум эффективного потенциала по параметру $a(\partial V / \partial a=0)$, получим условие $\alpha-n=a R$, или $n / 2 R=\alpha / 2 R-a / 2$. Преобразуем выражение для потенциала поля $A_{3}$ работы [51]

$$
A_{3}=\frac{\pi}{e a}=\frac{1}{2 e R}
$$

поскольку в работе [51] $a=2 \pi R$ - длина третьего измерения.

Обратимся к работе [36], преобразуем фазу Ааронова-Бома:

$$
\frac{2 \pi \phi_{\|}}{L}=\frac{2 \pi}{L} \frac{a A_{y} L}{2 \pi}=e A_{y} .
$$


Следовательно, в работе [51] фаза Ааронова-Бома равна

$$
e A_{3}=e \Delta=\frac{1}{2 R}
$$

Поскольку в нашей работе присутствует параметр фазового смещения $\alpha$, выражение для фазы будет несколько другим. Подставляя $n=1$ в условие минимума эффективного потенциала, получим

$$
\frac{1}{2 R}=\frac{1}{2}\left(\frac{\alpha}{R}-a\right)=\varphi,
$$

где $\varphi$ - искомая фаза Ааронова-Бома. Отсюда следует, что кинематический вклад для фермионов в виде фазы Ааронова-Бома уже включен в выражение для фермионной щели, получаемой как собственное значение массовой матрицы (см. [45])

$$
\lambda=\frac{1}{2}\left(\frac{\alpha}{R}-a\right) \pm \sqrt{\left(\frac{1}{2}\left(\frac{\alpha}{R}-a\right)\right)^{2}+|m|^{2}}=\varphi \pm \sqrt{\varphi^{2}+|m|^{2}} .
$$

В работе [51] также было показано, что состояние с наименьшей энергией соответствует фермионам с антипериодическими граничными условиями $A_{3} \equiv \Delta_{\min }=$ $\pi / e a=1 / 2 e R(a=2 \pi R)$, что соответствует полученному результату в нашей задаче: $a_{\min }=1 / 2 R\left(a=e A_{3}\right)$ при антипериодических граничных условиях $(\alpha=1 / 2)$, откуда получаем $A_{3}=1 / 2 e R$.

Отметим, что в рамках модели [36] также была рассчитана критическая константа связи, преобразуя которую, можно получить выражение, схожее с критической константой нашей задачи,

$$
G=\pi L\left\{\ln \left[\frac{\operatorname{ch}(L \Lambda)-\cos \left(2 \pi \phi_{\|}\right)}{1-\cos \left(2 \pi \phi_{\|}\right)}\right]\right\}^{-1},
$$

аналогично работе [36] с той лишь разницей, что в нашей модели присутствует фазовый параметр $\alpha$.

Аналогично работе [36] рассмотрим зависимость фермионной щели $\lambda R$ от фазы Ааронова-Бома $(\alpha-a R) / 2=\varphi R$ и убедимся в схожем поведении графиков. Поскольку уравнение для щели включает дополнительный параметр $m$, мы должны его зафиксировать. Уравнение щели $\partial V / \partial \sigma$ в нашем случае не решается аналитически, оценку для $m$ получим из графика зависимости $V_{\text {eff }} \Lambda^{-2}$ от $m \Lambda^{-1}$ при малых значениях поля $A_{3}$, поскольку при больших значениях поля фермионная щель будет иметь линейную зависимость от фазы Ааронова-Бома и $m R$ можно будет исключить как малый параметр. Из рис. 5 видно, что минимум эффективного потенциала достигается при $m R \simeq 0.1$, масса мало меняется, и, следовательно, ее можно зафиксировать. Отметим, что при нахождении $\lambda$ мы выбрали приближение $m R \ll 1$, и можно точно сказать, что это было обоснованно. Теперь построим зависимость $\lambda R$ от $\varphi R$ (см. рис. 6) и убедимся, что поведение щели в зависимости от фазы Ааронова-Бома получается схожим с полученным в работе [36]. Отличие нашей задачи заключается в наличии четырехфермионного взаимодействия, поэтому на рис. 6 присутствует вторая ветвь, где видно, что наряду с фермионной щелью, возрастающей с фазой Ааронова-Бома, генерируется также малая масса, много меньшая масс мод Калуцы-Клейна, что обсуждалось в разделе 2 настоящей работы. 

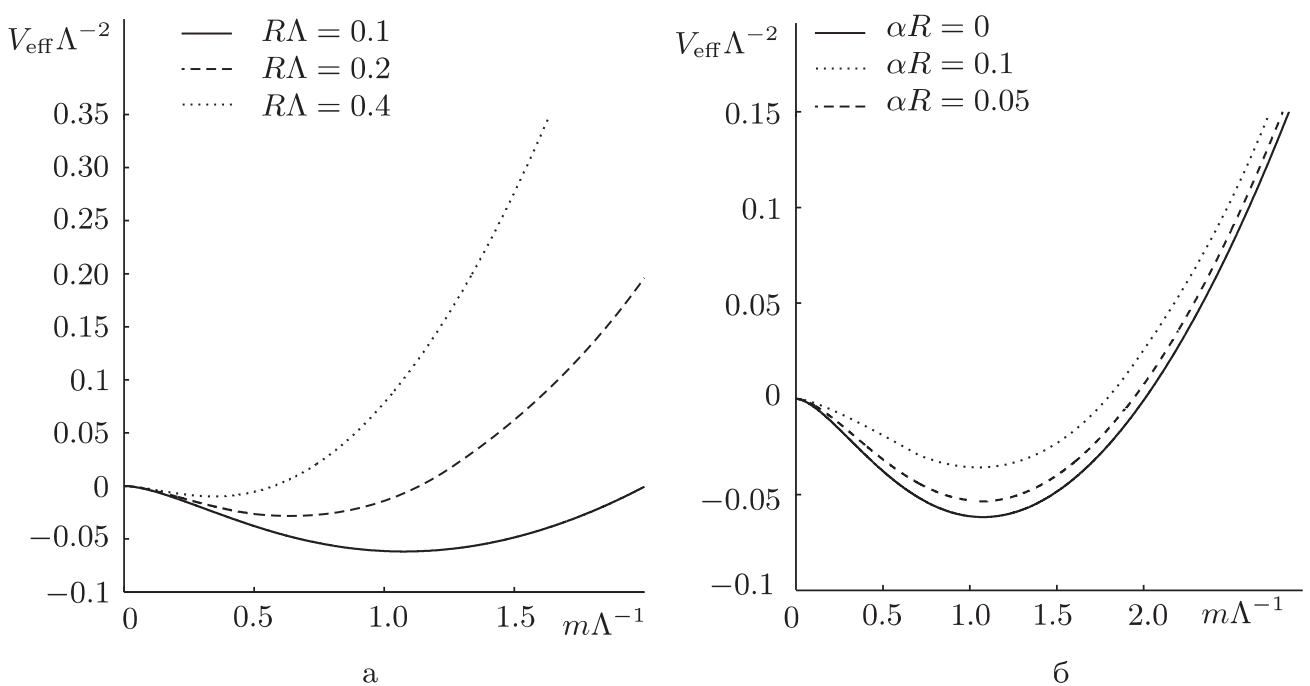

Рис. 5. Зависимость эффективного потенциала $V_{\text {eff }}$ от $m$ при различных значениях радиуса компактификации и $a=0, \alpha=0$ (а) и при $\alpha=0, R \Lambda=0.1$ и при различных малых значениях параметра поля $a$ (б). Минимум эффективного потенциала достигается при $m R \simeq 0.1$.

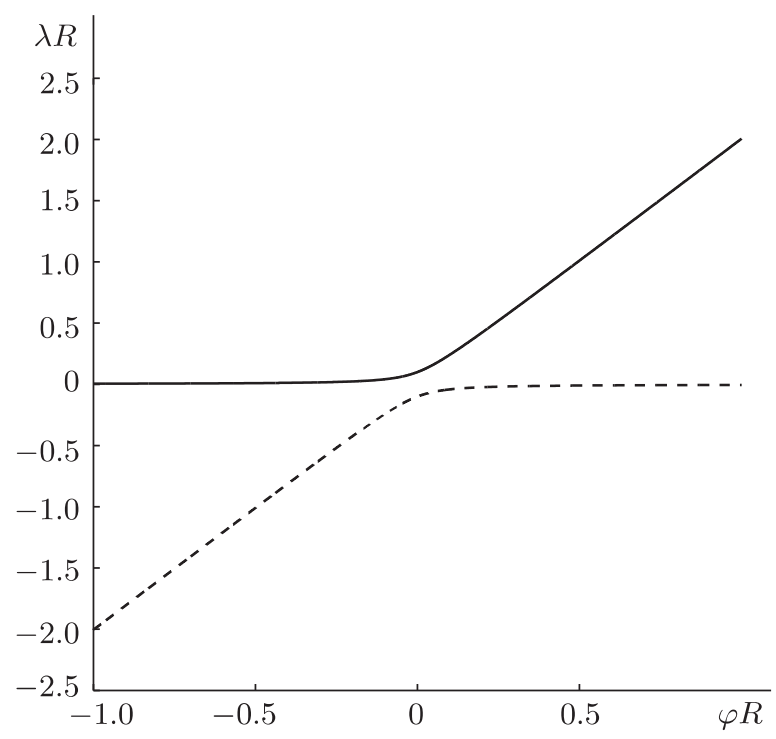

Рис. 6. Зависимость фермионного конденсата $\lambda R$ от фазы Ааронова-Бома $\varphi R=(\alpha-a R) / 2$.

3.2. Индуцированный ток. Рассмотрим эффект вакуумной поляризации, приводящий к возможности образования индуцированного тока в модели нанотрубки с лагранжианом, аналогичным лагранжиану из раздела 1 работы [52]. Данная 


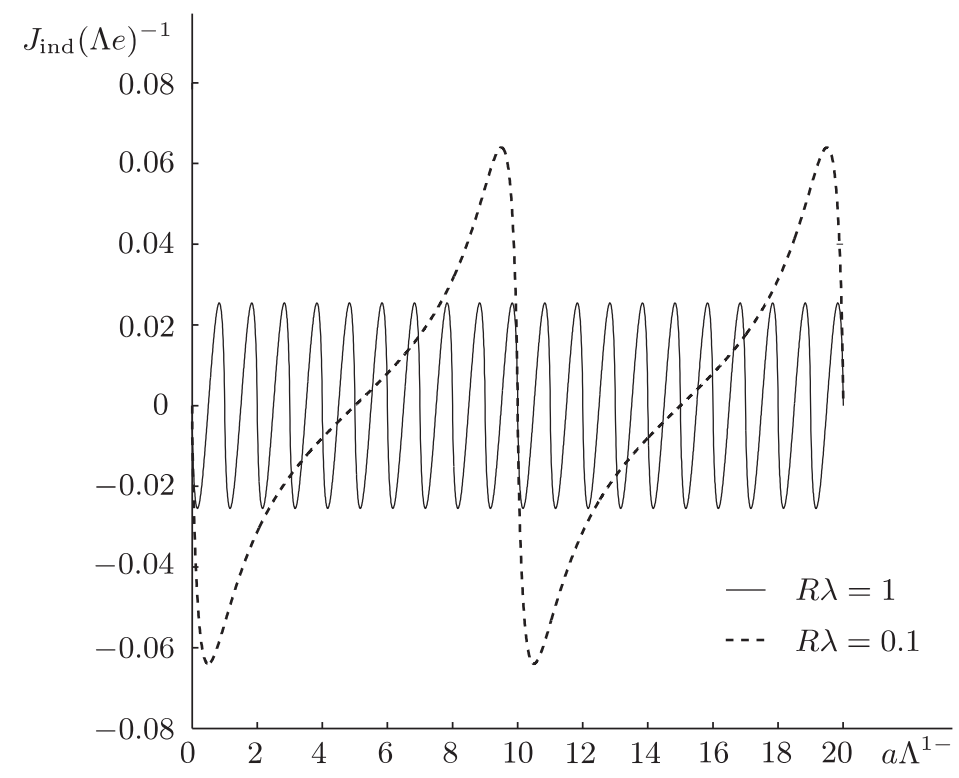

Рис. 7. Зависимость индуцированного тока от $а$ в критической точке $m=0$ при разных значениях радиуса компактификации $R$. Индуцированный ток обращается в нуль при $a R=n / 2$.

задача схожа с задачей, решаемой в работе [42], где рассматривалась вакуумная поляризация в модели графена в поле тонкого соленоида. Заметим, что именно нетривиальная топология, обусловленная вакуумными эффектами, проявляется в возникновении вакуумного тока. Индуцированный ток $J=\partial V_{\text {eff }} / \partial A_{3}$ направлен вдоль третьей координаты, и его можно найти с помощью формулы (17):

$$
\begin{aligned}
J_{\text {ind }}= & \frac{1}{2} e R \sin (2 \pi \nu) \int_{0}^{\infty} d x x\left(m^{4} \pi^{2} R^{2}-x^{2}\right) \times \\
& \times\left\{[\operatorname{ch}(2 \pi R x)-\cos (2 \pi \nu)] x^{2}+2 \pi R x|m|^{2} \operatorname{sh}(2 \pi R x)+\right. \\
& \left.\quad+[\operatorname{ch}(2 \pi R x)+\cos (2 \pi \nu)]\left(\pi R|m|^{2}\right)^{2}\right\}^{-1} .
\end{aligned}
$$

Заметим, что приведенный выше интеграл сходится, поэтому мы смогли распространить верхний предел $\Lambda$ до бесконечности.

Как видно из выражения (53), ток является периодической функцией переменной $\nu=e A_{3} R$ и обращается в нуль при $\nu=n / 2(n=0, \pm 1, \pm 2, \ldots)$, т. е. при $e \Phi / 2 \pi=n / 2$, где $\Phi=2 \pi R A_{3}$ - поток калибровочного поля, описываемого потенциалом $A_{3}$.

Интеграл (53) в критической точке $m=0$ в согласии с (18) упрощается и принимает вид

$$
J_{\text {ind }}=-\frac{1}{2} e R \sin (2 \pi \nu) \int_{0}^{\infty} \frac{x d x}{\operatorname{ch}(2 \pi R x)-\cos (2 \pi \nu)} .
$$

График зависимости индуцированного тока $J_{\text {ind }}$ от величины полевого параметpa $a=e A_{3}$ в случае $m=0$ показан на рис. 7 , где для удобства численных рас- 


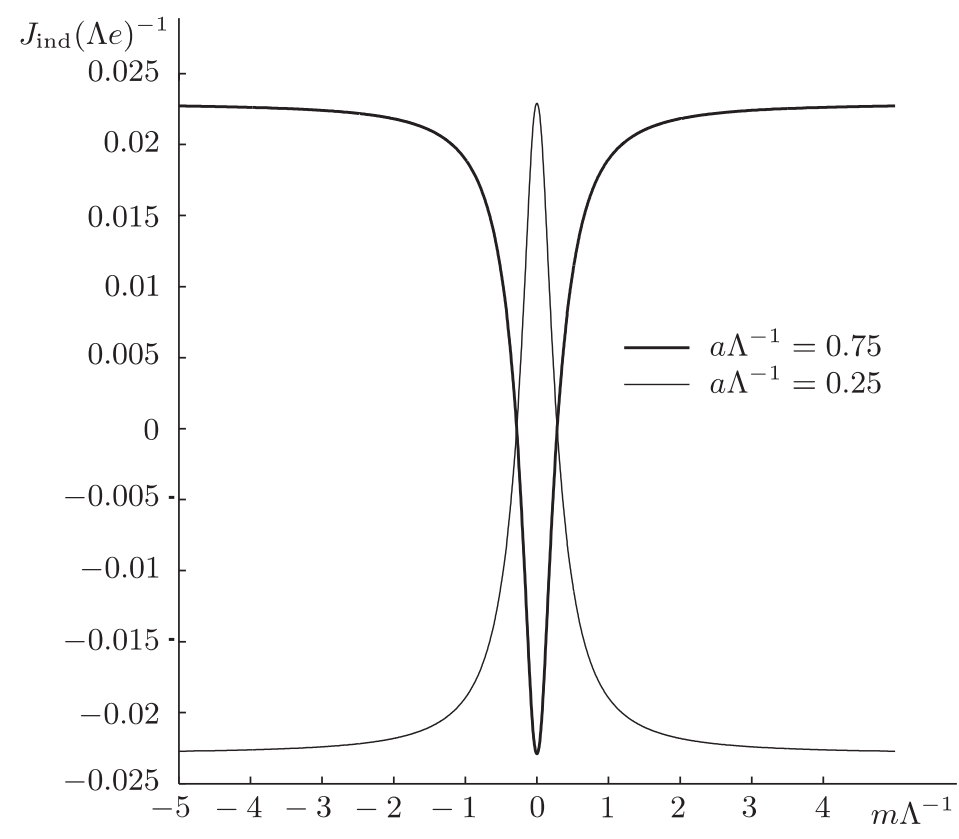

Рис. 8. Зависимость индуцированного тока от $m$ для постоянного значения радиуса компактификации при $R \Lambda=1$ и при разных $\nu$.

четов мы ввели безразмерные переменные с помощью параметра верхнего обрезания $\Lambda$ (см. (17)):

$$
\frac{J_{\text {ind }}}{e} \rightarrow \frac{J_{\text {ind }}}{e \Lambda}, \quad a \rightarrow a \Lambda^{-1}, \quad R \rightarrow R \Lambda .
$$

Заметим, что в таком случае величина $\nu=a R$ остается безразмерной.

Графики зависимости индуцированного тока $J_{\text {ind }}$ от величины $m$ показаны на рис. 8 и рис. 9 , где тоже использовались безразмерные переменные.

На рис. 8 показана зависимость тока $J_{\text {ind }}$ от величины $m$ при разных значениях $\nu=a R$ при одном и том же значении радиуса компактификации $R \Lambda=1$. Значения полевого параметра $a \Lambda^{-1}=0.25$ и $a \Lambda^{-1}=0.75$ выбраны с разных сторон от экстремума потенциала (18) $\nu=n / 2$ (в данном случае $\nu=0.5$ ), чтобы производная от потенциала имела разные знаки, а значит, ток $J_{\text {ind }}=\partial V_{\text {eff }} / \partial A_{3}$ имел разное поведение. На рис. 9 показана зависимость тока $J_{\text {ind }}$ от величины $m$ при разных значениях радиуса компактификации $R \Lambda$, но при одном и том же значении $\nu=a R$.

При тех значениях $\nu=e A_{3} R$, при которых величина $\tilde{\nu}(\tilde{\nu}=\nu-n$ при $\nu>0$ и $\tilde{\nu}=\nu+n$ при $\nu<0$, где $n$ - максимальное целое число, меньшее $|\nu|)$ принимает малые значения (эти точки соответствуют максимумам потенциала), ток будет равен

$$
\left.J_{\text {ind }}(\sigma, a)\right|_{\sigma=0} \approx \frac{e \tilde{\nu}}{4 \pi R} \ln \tilde{\nu}^{2}
$$

При тех значениях $\nu=e A_{3} R$, при которых величина $\tilde{\nu}=\nu-(2 n+1) / 2$ при $\nu>0$ и $\tilde{\nu}=\nu+(2 n+1) / 2$ при $\nu<0$, где $n-$ максимальное целое число, меньшее $|\nu|$, 


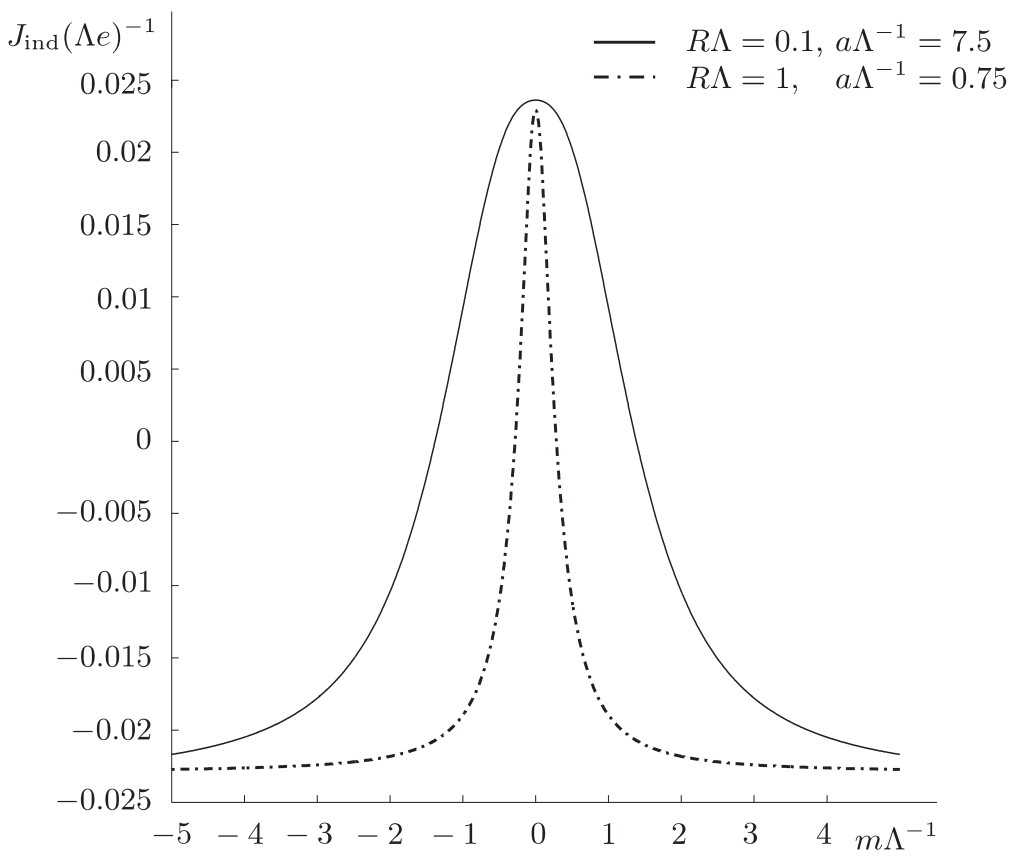

Рис. 9. Зависимость индуцированного тока от $m$ для постоянного значения $\nu$ при разных радиусах компактификации.

принимает малые значения (эти точки соответствуют минимумам потенциала), ток будет равен

$$
\left.J_{\text {ind }}(\sigma, a)\right|_{\sigma=0} \approx \frac{e \tilde{\nu}}{2 \pi R} \ln 2,
$$

что соответствует поведению кривой тока, приведенной на рис. 7 .

\section{4. ВЫВОДЫ}

В данной работе мы изучили процесс динамического образования массы фермионов в трехмерной модели с одним "дополнительным" измерением при взаимодействии двух типов фермионов, существующих в трехмерном пространстве и на двумерной бране, с учетом воздействия калибровочного поля $A_{3}$.

Если поле $A_{3}$ рассматривать как внешний параметр, то динамическая масса оказывается осциллирующей функцией (при $a \neq 0$ ) с амплитудой, уменьшающейся с ростом радиуса компактификации. При этом динамическая масса становится не зависящей от калибровочного поля при большом радиусе компактификации и стремится к постоянному значению (см. рис. 2а, б).

Если же рассматривать калибровочное поле $A_{3}$ как динамическую переменную, то экстремальное значение калибровочной переменной приводит к тривиальному значению константы связи, что изображено на рис. 4а. Следует отметить, что при условии (25) генерируемая масса (12) получается порядка $\lambda \sim 1 / 2 R$ при $(n=1)$, что равно ее значению при антипериодических граничных условиях $a=\alpha / R, \alpha=1 / 2$. Тем самым мы получаем массу, меньшую, чем массы мод Калуцы-Клейна $\lambda_{n}=n / R$. 
Если рассмотреть кривую константы связи (22), которая лежит немного выше критической величины $(m=0.1 \Lambda)$, то при изменении $R$ в области малых значений мы можем получить различные малые значения констант связи, а следовательно, и различные малые массы. Таким образом, в рамках данной модели существует возможность объяснить иерархию масс разных поколений частиц. При этом зависимость константы связи от малого радиуса компактификации дается формулой (35).

В работе была также исследована модель нанотрубки с размерностью $\mathbb{R}^{2} \times S^{1}$. В рамках данной задачи было установлено, что в выражение для фермионной щели кроме динамического вклада от конденсата $m$ из-за четырехфермионного взаимодействия входит кинематический вклад - фаза Ааронова-Бома. Отметим, что в нашей модели кинематический (топологический) вклад в фермионную щель был найден (в отличие от задачи в работе [36]) в результате решения задачи на минимум эффективного потенциала модели.

График зависимости фермионной щели от фазы Ааронова-Бома (рис. 6) демонстрирует, что в модели генерируются две массы. Сплошная линия показывает известную зависимость щели, как в работе [36]. Штриховая линия на рис. 6 показывает, что из-за наличия в модели четырехфермионного взаимодействия генерируется малая масса, много меньшая массы фермионов Калуцы-Клейна. В рамках настоящей задачи была рассчитана также критическая константа связи модели (см. выражение (52)).

Наконец, была рассмотрена возможность генерации индуцированного тока в $(2+$ 1)-мерной модели в “дополнительном" измерении. Мы показали, что именно эффект вакуумной поляризации приводит к возможности образования индуцированного тока. Отметим роль нетривиальной топологии, которая проявляется в возникновении вакуумного тока посредством вакуумных эффектов. Выражение для индуцированного тока дается формулой (53). Зависимость тока в критической точке $m=0$ от значения полевого параметра изображена на рис. 7. При значениях $\nu=e A_{3} R$, соответствующих максимуму эффективного потенциала, поведение индуцированного тока можно описать выражением (56), а при значениях, соответствующих минимуму потенциала, - выражением (57). Зависимость индуцированного тока от поля имеет периодический характер, и значение тока обращается в нуль при $\nu=n / 2\left(\nu=e A_{3} R\right.$, $n=0, \pm 1, \pm 2, \ldots)$.

Последующее исследование данной проблемы, по нашему мнению, может быть полезным в описании структуры и свойств более реалистических систем, таких как графен с линейными дефектами, нанотрубки и фуллерены.

\section{Список литературы}

[1] T. Kaluza, Sitzungsber. Preuss. Akad. Wiss. Berlin (Math. Phys.), 1921, 966-972.

[2] O. Klein, Z. Phys., 37:12 (1926), 895-906.

[3] R. Sundrum, TASI 2004 lectures: To the fifth dimension and back, arXiv: hep-th/0508134.

[4] Y. Hosotani, Phys. Lett. B, 126:5 (1983), 309-313.

[5] В. Ч. Жуковский, К. Г. Клименко, В.В. Худяков, Д. Эберт, Писъма в ЖЖТФ, 73:3 (2001), 137-142, arXiv: hep-th/0012256.

[6] В. Ч. Жуковский, К. Г. Клименко, В.В.Худяков, ТМФ, 124:2 (2000), 323-338.

[7] K. V. Zhukovskii, P. A. Eminov, Phys. Lett. B, 359:1-2 (1995), 155-158.

[8] К. В. Жуковский, П. А. Эминов, ЯФ, 59:7 (1996), 1265-1269. 
[9] В. Ч. Жууковский, А. С. Разумовский, К. В. Жуковский, Изв. вузов. Поволжский регион. Естественные науки, 2 (2003), 80-107, arXiv: hep-th/0402070.

[10] А. С. Вшивцев, Б. В. Магницкий, В. Ч. Жуковский, К. Г. Клименко, ЭЧА Я, 29:5 (1998), $1259-1318$.

[11] V. N. Rodionov, Phys. Rev. A, 75:6 (2007), 062111, 8 pp., arXiv: hep-ph/0702228.

[12] W. P. Su, J. R. Schrieffer, A. J. Heeger, Phys. Rev. Lett., 42:25 (1979), 1698-1701.

[13] K. S. Novoselov, A. K. Geim, S. V. Morozov, D. Jiang, Y. Zhang, S. V. Dubonos, I. V. Grigorieva, A. A. Firsov, Science, 306:5695 (2004), 666-669, arXiv: cond-mat/0410550.

[14] M. I. Katsnelson, Mater. Today, 10:1-2 (2007), 20-27.

[15] A. K. Geim, Science, 324:5934 (2009), 1530-1534, arXiv: 0906.3799.

[16] K. S. Novoselov, A. K. Geim, S. V. Morozov, D. Jiang, M. I. Katsnelson, I. V. Grigorieva, S. V. Dubonos, A. A. Firsov, Nature, 438:7065 (2005), 197-200, arXiv: cond-mat/0509330.

[17] A. H. Castro Neto, F. Guinea, N. M. R. Peres, K. S. Novoselov, A. K. Geim, Rev. Modern Phys., 81:1 (2009), 109-162, arXiv: 0709.1163.

[18] Y. Zhang, Y.-W. Tan, H. L. Stormer, P. Kim, Nature, 438:7065 (2005), 201-204, arXiv: cond-mat/0509355.

[19] K. S. Novoselov, Z. Jiang, Y. Zhang, S. V. Morozov, H. L. Stormer, U. Zeitler, J. C. Maan, G. S. Boebinger, P. Kim, A. K. Geim, Science, 315:5817 (2007), 1379-1379.

[20] P. R. Wallace, Phys. Rev., 71:9 (1947), 622-634.

[21] G. W. Semenoff, Phys. Rev. Lett., 53:26 (1984), 2449-2452.

[22] V.P. Gusynin, S. G. Sharapov, J. P. Carbotte, Internat. J. Modern Phys. B, 21:27 (2007), 4611-4658, arXiv: 0706.3016.

[23] A. H. Castro Neto, Selected topics in graphene physics, arXiv: 1004.3682.

[24] D. Ebert, V.Ch. Zhukovsky, E. A. Stepanov, J. Phys.: Condens. Matter, 26:12 (2014), $125502-125509$.

[25] D. Gross, A. Neveu, Phys. Rev. D, 10:10 (1974), 3235-3253.

[26] Y. Nambu, G. Jona-Lasinio, Phys. Rev., 122:1 (1961), 345-358.

[27] K. G. Klimenko, Z. Phys. C, 37:3 (1988), 457-463.

[28] B. Rosenstein, B. J. Warr, S. H. Park, Phys. Rev. D, 39:10 (1989), 3088-3092.

[29] D. Ebert, M. K. Volkov, Phys. Lett. B, 272:1-2 (1991), 86-90.

[30] H. Caldas, O. Rudnei, Phys. Rev. B, 80:11 (2009), 115428, 9 pp., arXiv: 0907.0723.

[31] J. E. Drut, D. T. Son, Phys. Rev. B, 77:7 (2008), 075115, 5 pp., arXiv: 0710.1315.

[32] H. Caldas, Nucl. Phys. B, 807:3 (2009), 651-658.

[33] F. de Juan, A. Cortijo, M. A. H. Vozmediano, Phys. Rev. B, 76:16 (2007), 165409, 9 pp.

[34] M. A. H. Vozmediano, M. I. Katsnelson, F. Guinea, Phys. Rep., 496:4-5 (2010), 109-148, arXiv: 1003.5179 .

[35] J. González, F. Guinea, M. A. H. Vozmediano, Nucl. Phys. B, 406:3 (1993), 771-794.

[36] A. V. Gamayun, E. V. Gorbar, Phys. Lett. B, 610:1-2 (2005), 74-79, arXiv: hep-ph/ 0411157.

[37] E. J. Ferrer, V. de la Incera, Photons and fermions in spacetime with a compactified spatial dimension, arXiv: hep-ph/0408229.

[38] D. Y. Song, Phys. Rev. D, 48:8 (1993), 3925-3928.

[39] Yu. A. Sitenko, Phys. Rev. D, 60:12 (1999), 125017, 13 pp.

[40] Y. Aharonov, D. Bohm, Phys. Rev. (2), 115:3 (1959), 485-491.

[41] В. Г. Кадышевский, В. Н. Родионов, ТМФ, 136:3 (2003), 517-528.

[42] R. Jackiw, A. I. Milstein, S.-Y. Pi, I. S. Terekhov, Phys. Rev. B, 80:3 (2009), 033413, 3 pp.

[43] H. Abe, H. Miguchi, T. Muta, Modern Phys. Lett. A, 15:6 (2000), 445-454, arXiv: hep-ph/0002212.

[44] D. Ebert, V. Ch. Zhukovsky, A. V. Tyukov, Modern Phys. Lett. A, 25:35 (2010), 2933-2945. 
[45] V. Ch. Zhukovsky, E. A. Stepanov, Phys. Lett. B, 718:2 (2012), 597-602.

[46] И. П. Волобуев, В. Г. Кадышевский, М. Д. Матвеев, Р. М. Мир-Касимов, ТМФ, 40:3 (1979), 363-372.

[47] W. Bietenholz, A. Gfeller, U. J. Wiese, JHEP, 10 (2003), 018, 24 pp., arXiv: hep-th/ 0309162

[48] В. Ч. Жуковский, Е.А. Степанов, Вестн. Моск. ун-та. Сер. 3. Физ. Астрон., 2012, № $1,58-64$.

[49] R. G. Chambers, Phys. Rev. Lett., 5:1 (1960), 3-5.

[50] N. Osakabe, T. Matsuda, T. Kawasaki, J. Endo, A. Tonomura, S. Yano, H. Yamada, Phys. Rev. A, 34:2 (1986), 815-822.

[51] E. J. Ferrer, V. de la Incera, A. Romeo, Phys. Lett. B, 515:3-4 (2001), 341-347.

[52] В. Ч. Жуковский, Е. А. Степанов, Вестн. МГУ. Сер. 3. Физ. Астрон., 2014, № 2, 36-42.

Поступила в редакцию 10.06.2014 